\title{
Multi-Exponential Relaxation Times Maps Reconstruction and Unsupervised Classification in Magnitude Magnetic Resonance Imaging
}

\author{
Christian EL HAJJ \\ Irstea and LS2N \\ Rennes And Nantes France \\ Christian.s.hajj@gmail.com
}

\author{
Saïd MOUSSAOUI \\ LS2N, UMR CNRS 6004 \\ Centrale Nantes, Nantes, France \\ Saïd.Moussaoui@ec-nantes.fr
}

\author{
Guylaine COLLEWET \\ Irstea \\ Rennes, France \\ Guylaine.Collewet@irstea.fr
}

\author{
Maja MUSSE \\ Irstea \\ Rennes, France \\ Maja.Musse@irstea.fr
}

\begin{abstract}
In clinical and biological applications of $T_{2}$ relaxometry, a multi-exponential decay model proved to be representative of the relaxation signal inside each voxel of the MRI images. However, estimating and exploiting the model parameters for magnitude data is a large-scale ill- posed inverse problem. This paper presents a parameter estimation method that combines a spatial regularization with a Maximum-Likelihood criterion based on the Rician distribution of the noise. In order to properly carry out the estimation on the image level, a MajorizationMinimization approach is implemented alongside an adapted non-linear least-squares algorithm. We propose a method for exploiting the reconstructed maps by clustering the parameters using a K-means classification algorithm applied to the extracted relaxation time and amplitude maps. The method is illustrated on real MRI data of food sample analysis.
\end{abstract}

Index Terms-MRI, multi-exponential model, MaximumLikelihood, Majorization-Minimization, K-means.

\section{INTRODUCTION}

In MRI transverse relaxation $\left(T_{2}\right)$ relaxometry, a series of $T_{2}$-weighted images are acquired at a fixed sampling rate using a multiple spin echo (Multi-SE) technique. In most approaches, a mono-exponential decay curve is fitted to the measured signals, and one $T_{2}$ per voxel is estimated (time constants of the decay curve) alongside their corresponding amplitudes $A_{0}$ [1]. However, in many cases a multiexponential decay curve can provide more relevant information on the micro-structure or composition of the tissue. Actually, since the size of an MRI voxel is of the order of $1 \mathrm{~mm}^{3}$, it may contain several different components such as lipids and water, or several kinds of water pools with different $T_{2}$. The latter case is typically observed when analyzing MRI data of plants. Multi-exponential $T_{2}$ and $A_{0}$ maps can be used to access information about water status and distribution at the sub-cellular level. This is useful for example for the assessing of fruit characteristics in the monitoring of the post-harvest ripening of tomato fruit [10]. The Multi-exponential behavior is attributed to different water pools corresponding to the compartments of the plant cell (vacuole, cytoplasm and wall). The same situation occurs for myelin water fraction in the brain [9],

Thanks to Region Bretagne, École centrale Nantes, Irstea Rennes and GDR ISIS for funding. where three major components are used to represent the water trapped between the myelin, the intra/extra-cellular water and the cerebro-spinal fluid. The multi-exponential aspect of the measured decay signal can be described by a continuous $T_{2}$ distribution, or by limiting the number of exponential decay curves inside each voxel to a small discrete number equal to the number of components. In this study, the expected number of components is considered as known and is of low value (triexponential model).

The noise in the MRI data is considered to follow an independent and identically distributed Gaussian noise in the complex domain. However, the phase of the complex data is both time and space dependent due to various uncontrollable factors related to the acquisition process. Thus, estimating the parameters directly in the complex domain proves to be a challenging problem. The common approach is to carry out the parameters estimation from the magnitude of the measured signals. This non-linear transformation, in the case of single coil acquisitions [5], results in a Rician distributed noise. Rician noise can be approximated by a Gaussian distribution for high signal to noise ratio (SNR). However, in relaxometry, the SNR of the acquired data is critically low, particularly at the end of the relaxation curve. If the Rician noise distribution is not properly accounted for, it leads to a bias on the estimated parameters [11].

A Maximum Likelihood (ML) estimator based on the Rician probability density function is implemented in order to reduce the estimation bias caused by the Rician distribution [13]. Moreover, in order to stabilize the algorithm for low SNR values, a spatial regularization approach is implemented by taking advantage of low voxel to voxel variability at different image structures [6], [8]. Due to the large amount of memory needed to directly implement the spatial regularization on the image level, it has been carried out either by dividing the image into overlapping patches [8] or by carrying the estimation on regions of interest.

The exploitation of the estimated parameters proves to be a complicated process due to the large amount of information in the reconstructed maps. A step of data classification in the space formed by the estimated parameters is suggested in 
order to extract a distribution of the estimated $T_{2}$ parameters at different spatial regions of the image.

In this paper, an efficient penalized ML algorithm is presented in order to carry out the estimation on the whole image at once and obtain voxel level information [2]. The first feature of the algorithm is to estimate all the image parameters simultaneously using a Majorization-Minimization (MM) approach leading to a quadratic majorant function to be minimized with an adapted Levenberg-Marquardt (LM) algorithm. The particularity is to compute a maximum step-size that guarantees the parameters positivity and to adopt a step search approach using a backtracking technique based on the Armijo line search to find a step-size that ensures the convergence of the algorithm. The main contribution of this paper is to propose an appraoch allowing to exploit the extracted parameters. It is based on clustering the estimated parameters using a non-supervised classification approach based on the K-means algorithm. Tests were conducted on simulated phantom and on experimental MRI images of a tomato. The performances are illustrated through numerical and visual results.

\section{PARAMETERS ESTIMATION}

Magnitude MRI data, measured with a Multi-SE sequence, are composed of $N_{t}$ images. The fixed sampling time between two successive images is given by $\Delta T E$. For a multiexponential decay signal with $N_{c}$ components inside voxel $j$, the measured signal $m_{j}(\tau)$, can be fitted by a predicted signal model $A_{j}\left(\tau, \boldsymbol{\theta}_{\boldsymbol{j}}\right)$ expressed by :

$$
A_{j}\left(\tau, \boldsymbol{\theta}_{\boldsymbol{j}}\right)=\sum_{c=1}^{N_{c}} A_{0_{(c, j)}} e^{-\frac{\tau}{T_{2}(c, j)}}, \quad \text { for } \tau=\tau_{1}, \ldots, \tau_{N_{t}}
$$

where $\boldsymbol{\theta}_{\boldsymbol{j}}=\left[\begin{array}{lllll}A_{0_{(1, j)}}, T_{2_{(1, j)}} \ldots & A_{0_{\left(N_{c}, j\right)}}, T_{2_{\left(N_{c}, j\right)}}\end{array}\right]$ is the vector of unknown parameters of length $N_{p}=2 N_{c}$.

\section{A. Problem statement}

In magnitude MRI data with a sufficiently high SNR, the noise distribution can be approximated by a Gaussian distribution. However, at low SNR the relaxation data statistics follows a Rician distribution model. Thus, in order to precisely estimate the parameters, the Rician probability density function $P_{R}$ should be taken into account. This leads to a Maximum Likelihood estimator that can be solved by maximizing the following likelihood function :

$$
\begin{array}{r}
L(\boldsymbol{m}, \boldsymbol{\theta})=\prod_{j=1}^{N_{v}} \prod_{\tau=\tau_{1}}^{\tau_{N_{t}}} P_{R}\left(m_{j}(\tau) \mid A_{j}\left(\tau, \boldsymbol{\theta}_{\boldsymbol{j}}\right), \sigma\right)= \\
\prod_{j=1}^{N_{v}} \prod_{\tau=\tau_{1}}^{\tau_{N_{t}}} \frac{m_{j}(\tau)}{\sigma^{2}} e^{-\left[\frac{m_{j}(\tau)^{2}+A_{j}\left(\tau, \boldsymbol{\theta}_{\boldsymbol{j}}\right)^{2}}{2 \sigma^{2}}\right]} I_{0}\left(D_{j \tau}\right),
\end{array}
$$

where $N_{v}$ is the number of voxels, $\boldsymbol{m}$ is a vector containing the measurement at all the voxels of the MRI image, $\boldsymbol{\theta}=$ $\left[\begin{array}{lll}\boldsymbol{\theta}_{\mathbf{1}} & \ldots & \boldsymbol{\theta}_{\boldsymbol{N}_{\boldsymbol{v}}}\end{array}\right], I_{0}(\cdot)$ is the zeroth order modified Bessel function of the first kind, $D_{j \tau}=\frac{m_{j}(\tau) A_{j}\left(\tau, \boldsymbol{\theta}_{j}\right)}{\sigma^{2}}$, and $\sigma^{2}$ is the variance of the Gaussian noise. By taking the $\log$ of the likelihood function and reformulating, minimizing the likelihood function in (2) comes down to minimizing the following ML criterion :

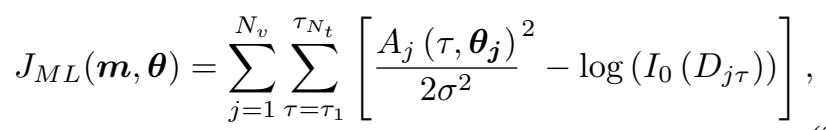

Estimating the high number of parameters under low SNR conditions is an ill posed problem. The spatial regularity at different image structure is accounted for by adding a penalizing function to the $J_{M L}$ criterion. For each voxel $j$, a penalty function $\psi$ is computed such that high differences between parameters belonging to a predefined neighboring region $V_{j}$ are penalized :

$$
R(\boldsymbol{\theta})=\sum_{p=1}^{N_{p}} \beta(p) \sum_{j=1}^{N_{v}} \sum_{i \in V_{j}} \psi\left(\theta_{j}(p)-\theta_{i}(p)\right)
$$

$\boldsymbol{\beta}$ is the vector that controls the weight attributed to the spatial regularization. An L1-L2 penalty function $\psi$ was used in order to minimize the inter-tissue blurring [7]. The penalized ML (PML) criterion to be minimized is thus given by :

$$
F(\boldsymbol{m}, \boldsymbol{\theta})=J_{M L}(\boldsymbol{m}, \boldsymbol{\theta})+R(\boldsymbol{\theta})
$$

\section{B. Proposed algorithm}

The PML criterion does not have an analytical solution thus, an iterative approach must be adopted. In order to minimize the $F(\boldsymbol{m}, \boldsymbol{\theta})$ criterion, two main challenges must be resolved; the first one is the non-convexity of the $J_{M L}(\boldsymbol{m}, \boldsymbol{\theta})$ criterion and the second one is the voxel-wise dependency of the solution due to the regularization penalty function.

The optimization algorithm is based on a MM approach. It allows the minimization of a non quadratic, non convex and multidimensional criterion by transforming it into a separable quadratic criterion easier to minimize. Firstly, the strictly concave $-\log \left(I_{0}(\cdot)\right)$ function is majorized by its tangent :

$-\log \left(I_{0}\left(D_{j \tau}^{(k)}\right)\right)-\frac{m_{j}(\tau)}{\sigma^{2}} R\left(D_{j \tau}^{(k)}\right)\left(A_{j}\left(\tau, \boldsymbol{\theta}_{j}\right)-A_{j}\left(\tau, \boldsymbol{\theta}_{j}^{(\boldsymbol{k})}\right)\right)$

where $\theta_{j}^{(k)}$ is the the vector of the estimated parameters at the $k$ th iteration of the MM algorithm and $R(\cdot)=\frac{I_{1}(\cdot)}{I_{0}(\cdot)}$ where $I_{1}(\cdot)$ is the first order modified Bessel function of the first kind. The obtained quadratic ML criterion to be minimized at each step $k$ of the MM algorithm is given by [12] :

$Q_{M L}\left(\boldsymbol{m}, \boldsymbol{\theta}^{(k)}\right)=\frac{1}{2 \sigma^{2}} \sum_{j=1}^{N_{v}} \sum_{\tau=\tau_{1}}^{\tau_{N_{t}}}\left(m_{j}(\tau) R\left(D_{j \tau}^{(k)}\right)-A_{j}\left(\tau, \boldsymbol{\theta}_{j}\right)\right)^{2}$.

Secondly, Erdogan and Fesslers's [3] method is used to construct a majorant function that establishes a voxel-wise variables separability. Accordingly, for each iteration $k$ of the MM algorithm, the regularization function is majorized by :

$$
\begin{aligned}
\psi\left(z_{j}-z_{i}\right) \leq \frac{1}{2} \psi\left(2 z_{j}\right. & \left.-\left(z_{j}^{(k)}+z_{i}^{(k)}\right)\right) \\
+ & \frac{1}{2} \psi\left(2 z_{i}-\left(z_{j}^{(k)}+z_{i}^{(k)}\right)\right) .
\end{aligned}
$$


The obtained majorant criterion of the $F(\boldsymbol{m}, \boldsymbol{\theta})$ is given by $Q_{P M L}\left(\boldsymbol{m}, \boldsymbol{\theta}^{(\boldsymbol{k})}\right)=Q_{M L}\left(\boldsymbol{m}, \boldsymbol{\theta}^{(\boldsymbol{k})}\right)+Q_{r e g}\left(\boldsymbol{\theta}^{(\boldsymbol{k})}\right)$ with :

$$
\begin{aligned}
& Q_{\text {reg }}\left(\boldsymbol{\theta}^{(k)}\right)= \\
& \quad \sum_{p=1}^{N_{p}} \beta(p) \sum_{j=1}^{N_{v}} \sum_{i \in V_{j}} \psi\left(2 \theta_{j}(p)-\left(\theta_{j}^{(k)}(p)+\theta_{i}^{(k)}(p)\right)\right)
\end{aligned}
$$

The minimization of $Q_{P M L}\left(\boldsymbol{m}, \boldsymbol{\theta}^{(\boldsymbol{k})}\right)$ criterion is performed by a Levenberg-Marquardt (LM) algorithm witch is suited for solving non-linear least-squares problems. At iteration $l$ of the LM algorithm, the parameters are updated in a direction $\boldsymbol{d}_{L M}$ depending on the residual $\boldsymbol{r}$ and the Jacobian matrix $\boldsymbol{J}$ :

$$
\boldsymbol{d}_{\boldsymbol{L} M}^{(l)}=\left[\boldsymbol{J}^{(l)^{T}} \boldsymbol{J}^{(l)}+\boldsymbol{\lambda}^{(l)}\right]^{-1} \boldsymbol{J}^{(l)^{T}} \boldsymbol{r}^{(l)}
$$

$\boldsymbol{r}$ is expressed such that $Q_{P M L}\left(\boldsymbol{m}, \boldsymbol{\theta}^{(\boldsymbol{k})}\right)=\frac{1}{2}\|\boldsymbol{r}\|_{2}^{2} \cdot \boldsymbol{\lambda}$ is a diagonal matrix that contains the LM parameters for each voxel. In order to ensure the physical coherence of the estimated parameters, a positivity constraint is imposed. In order to ensure a sufficient decrease of the criterion, the stepsize is computed using a backtracking technique based on the Armijo condition bounded by a maximum step-size that guarantees the positivity of the parameters. The proposed MMPML algorithm is summarized in algorithm 1.

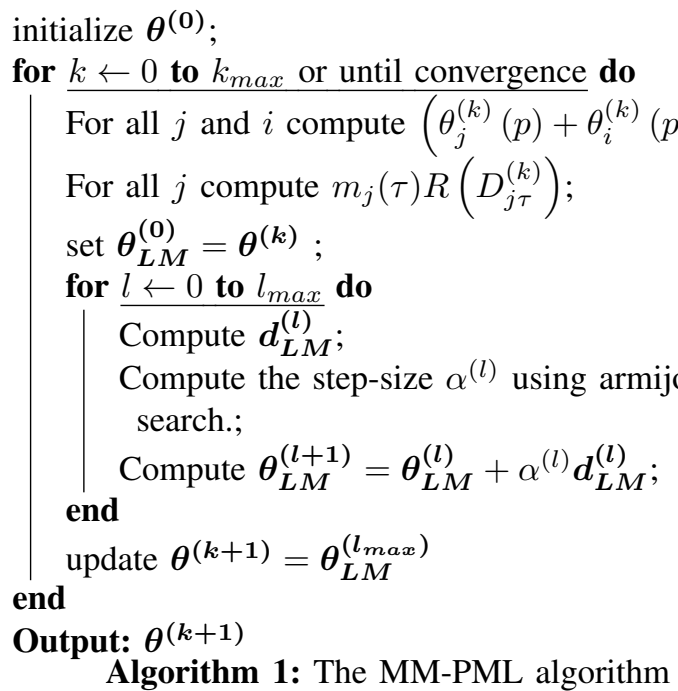

The algorithm is said to have converged if the value of the gradient is smaller than a tolerance value gtol or if the difference between the objective function at step $k+1$ and $k$ is smaller than a tolerance value ftol :

$$
\frac{F\left(\boldsymbol{m}, \boldsymbol{\theta}^{(\boldsymbol{k}+\mathbf{1})}\right)-F\left(\boldsymbol{m}, \boldsymbol{\theta}^{(\boldsymbol{k})}\right)}{F\left(\boldsymbol{m}, \boldsymbol{\theta}^{(\boldsymbol{k}+\mathbf{1})}\right)} \leq \text { ftol } .
$$

C. Classification of the results based on the estimated parameters

The reconstructed multi-exponential maps contain information that can be exploited in order to classify the image composition into coherent regions and extract the corresponding $T_{2}$ distribution. To our knowledge, this problem has not yet

\begin{tabular}{|c|c|c|c|c|c|c|}
\hline & $A_{01}$ & $T_{21}$ & $A_{02}$ & $T_{22}$ & $A_{03}$ & $T_{23}$ \\
\hline$\bullet$ & 95 & 88 & 459 & 356 & 1015 & 716 \\
\hline$\bullet$ & 96 & 76 & 410 & 433 & 1024 & 870 \\
\hline$\bullet$ & 71 & 50 & 339 & 218 & 908 & 627 \\
\hline$\bullet$ & 109 & 78 & 375 & 303 & 1004 & 685 \\
\hline$\bullet$ & 108 & 50 & 482 & 202 & 756 & 508 \\
\hline
\end{tabular}

Fig. 1. $T_{2}$ and $A_{0}$ values used for generating the phantom.

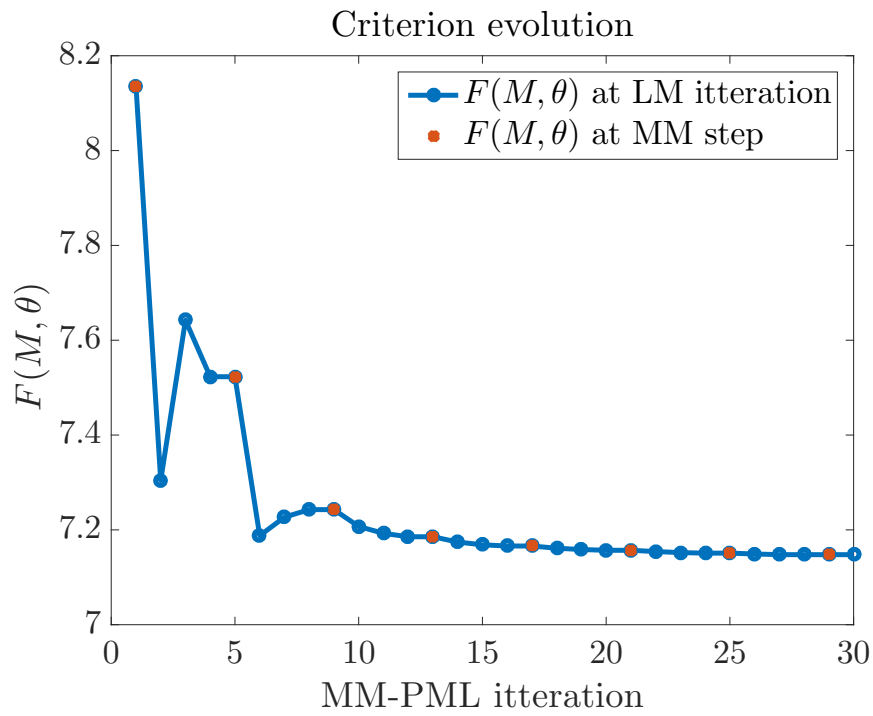

Fig. 2. The evolution of the PML criterion (in blue) across the external MM steps (orange points) and the internal LM steps (blue points)

been treated in the context of MRI relaxometry. Actually, Each voxel $j$ of the image have $N_{p}$ estimated parameter that can be considered as features. The K-means clustering algorithm was applied [4] to the whole image as it is an unsupervised method of classification. The number of classes is chosen visually, such that, by adding another class, two classes with very close $T_{2}$ distributions will be obtained. Finally, the results of the classification with the corresponding $T_{2}$ distribution inside each class are shown.

\section{MATERIALS}

Both the MM-PML algorithm and the K-means clustering are tested on simulated and experimental MRI data.

\section{A. Simulated data}

A circular phantom was constructed on an image of $128 \times$ 128 voxels, with an outside ring and 9 inner disks of different radii. At each part of the phantom, a different tri-exponential model was generated. $T_{2}$ and $A_{0}$ values that are close to parameters typically found in tomato fruits were used as seen in Figure 1. Then, a Rician noise was added with a variance of $\sigma^{2}=49$ in order to obtain a SNR value close to that found in experimental MRI data (212) $\left(S N R=\frac{\sum_{j=1}^{N} m_{j}\left(\tau_{1}\right)}{\sigma N_{v}}\right)$. 

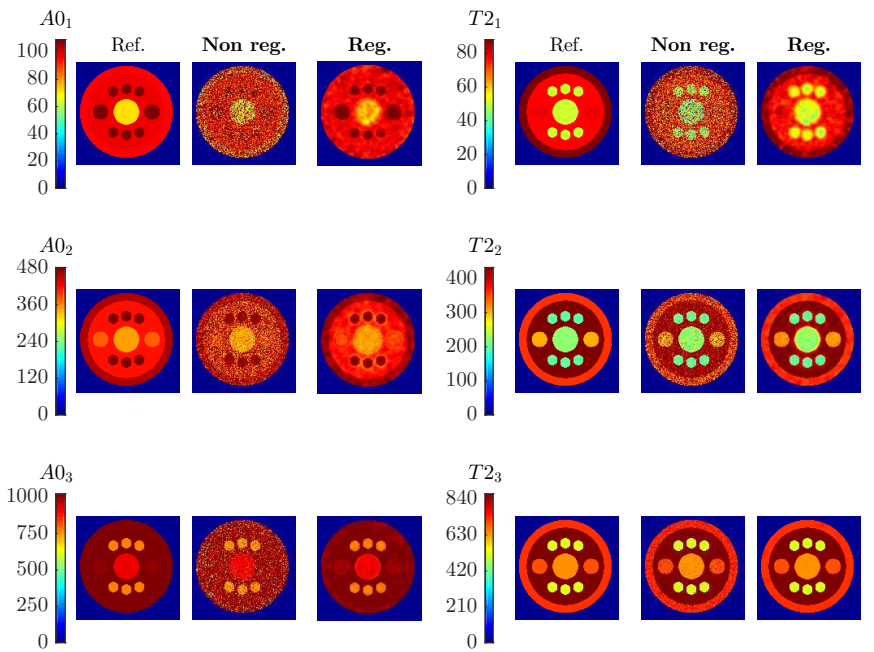

Fig. 3. Reference (first columns) and estimated $A_{0}$ and $T_{2}$ maps on the phantom using a non-regularized (second columns) and the proposed regularized algorithm (third columns).
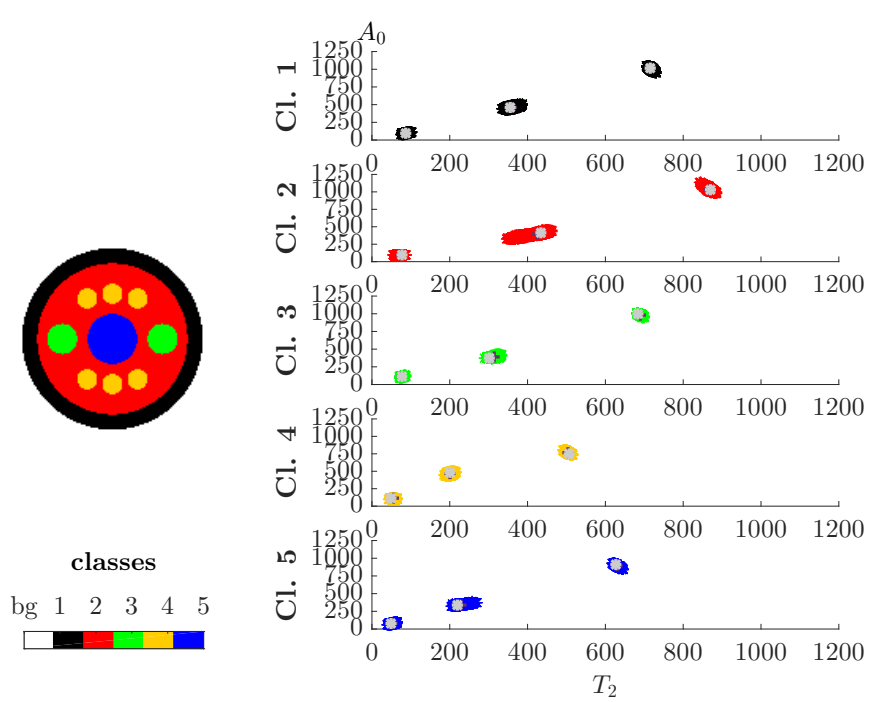

Fig. 4. Spatial partitioning of the 5 classes identified by the K-means algorithm and the joint distribution of parameters (T2;A0).The violet crosses represent the position of the estimated centroids. The gray astroïdes represent the real values used for simulation.

\section{B. Experimental data}

For the experimental MRI settings, a Multi-SE sequence was used on a $1.5 \mathrm{~T}$ MRI scanner (Magnetom, Avanto, Siemens, Erlangen, Germany), with inter-echo spacing $(\Delta \mathrm{TE})$ of $6.5 \mathrm{~ms}$, bandwidth of $260 \mathrm{~Hz} /$ pixel, 512 echoes per echo train and a repetition time of $10 \mathrm{~s}$. The median planes of fruit (transverse section at middle height of fruit) were imaged with a total of $128 \times 128$ voxels and a slice thickness of $5 \mathrm{~mm}$, resulting in voxel size of $1.19 \times 1.19 \times 5 \mathrm{~mm}^{3}$. The reference maps where acquiring with 32 scans in order to obtain higher SNR (687), and to verify the stability of the algorithm at the lowest SNR (145), the same images with only one scan were acquired.
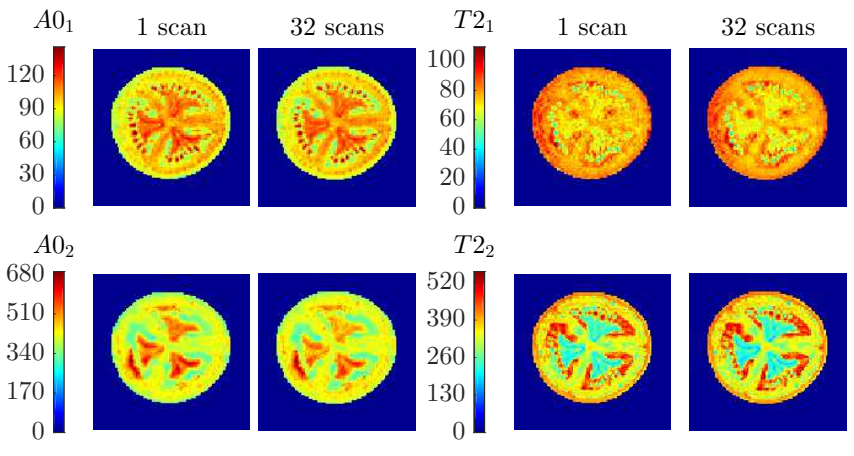

$T 2_{2}$
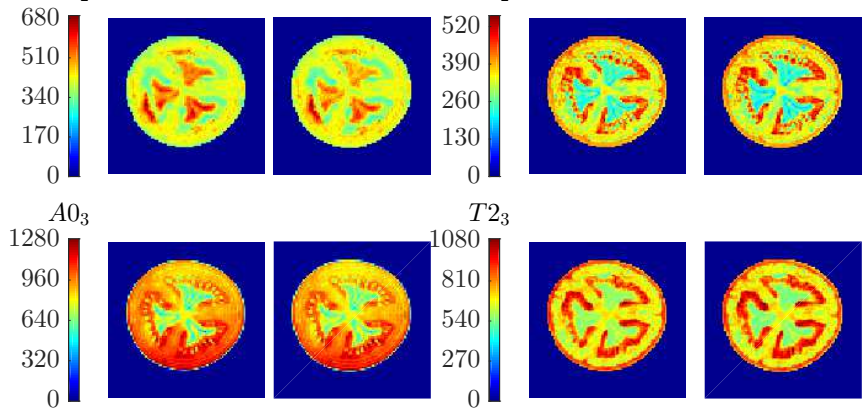

Fig. 5. $A_{0}$ and $T_{2}$ maps reconstructed from MRI images of a tomato acquired with 32 scans and 1 scan.
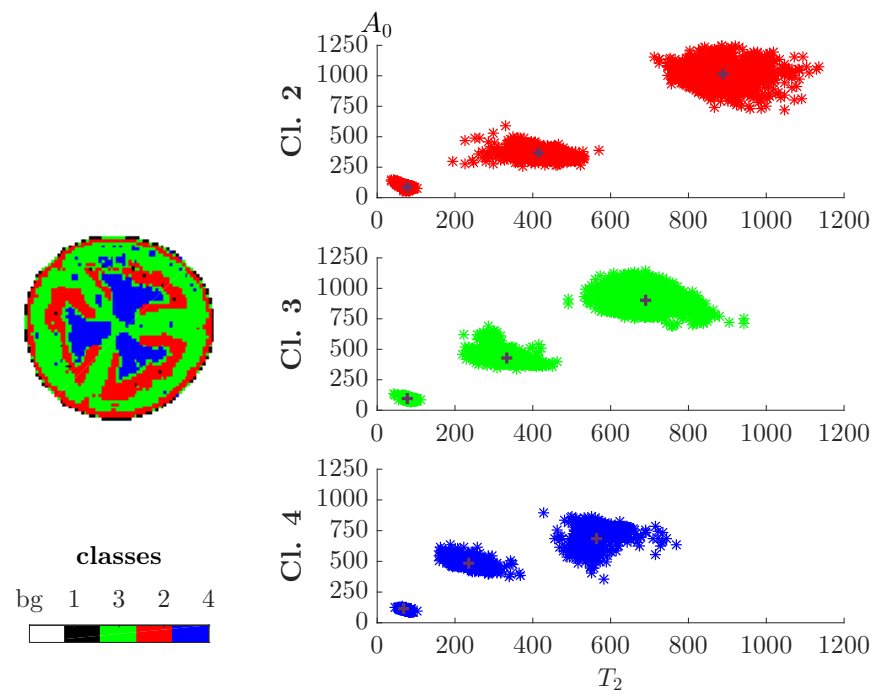

Fig. 6. Spatial partitioning of the 5 classes identified by the K-means algorithm and the joint distribution of parameters (T2;A0). The violet crosses represent the position of the estimated centroids.

\section{Settings}

In order to implement the regularization, the penalizing function $\psi$ and the penalizing weight vector $\boldsymbol{\beta}$ must to be chosen. The choice of the penalizing function affects the intertissues smoothness, whilst the penalizing weight affects the degree of smoothness on the whole image. In this study, an L1-L2 penalizing function was used with a weight $\boldsymbol{\beta}$ chosen by trial and error so as to preserve a good separation between edges. The algorithm stopping criteria gtol and ftol were both set to $10^{-6}$. Numerical results were computed using the normalized root mean square error (NRMSE) that was computed as follows :

$$
N R M S E=100 \sqrt{\frac{1}{N_{v} N_{p}} \sum_{j=1}^{N_{v}} \sum_{p=1}^{N_{p}} \frac{\left(\theta_{j}(p)-\theta_{j}^{*}(p)\right)^{2}}{\theta_{j}^{*}(p)^{2}}}
$$


with $\boldsymbol{\theta}_{j}^{*}$ the reference parameters at voxel $j$.

\section{RESULTS}

In Figure 2 the value of the $F(\boldsymbol{m}, \boldsymbol{\theta})$ criterion is shown at every step of the MM algorithm. By applying the LM algorithm to minimize surrogate criterion, a descent of the initial criterion is guaranteed, thus validating the correctness of the choice of the majorant functions.

\section{A. Simulated data}

In Figure 3 the results of applying the MM-PML on the phantom are shwon and are compared to a non-regularized version of the algorithm $(\boldsymbol{\beta}=0)$. Using the regularized algorithm, the maps are reconstructed with satisfying edge preserving which allows us to visually distinguish between the different structures even in low contrast situations. By adopting the spatial regularization, the noise of the reconstructed maps was reduced and the contrast between different image regions was more clearly pronounced. Numerically, a low NRMSE/voxel/parameter was obtained for the regularized version $4.47 \%$ whereas for the non regularized version we obtained a value of $22.85 \%$. This further validates the importance of the regularization on reducing the estimation error.

In Figure 4, the classification algorithm was applied to the results of the parameters obtained with the MM-PML algorithm. The different voxels were associated to their right class with a precision of a $100 \%$. Furthermore, the distribution of $T_{2}$ values inside each class are shown and the centroids estimated using the k-means algorithm proved to be very close to the true values used for simulating the phantom.

\section{B. Experimental data}

Figure 5 shows that images reconstructed from low and high SNR data are quite similar for the different parameters, thus validating the robustness of the method to different noise levels. Furthermore, a low NRMSE equal to $5.56 \%$ was obtained. This shows that even with low SNR the algorithm converged to values close to those estimated from high SNR data which will allow us to reduce the acquisition time by scanning the fruit with only one repetition. Figure 6 shows the results of the k-means algorithm and the distribution of the $T_{2}$ values inside each identified class. Three different tissues (out of the anticipated five) of the tomato are well identified using the proposed classification technique and are attributed to a class with different $T_{2}$ distribution. The red class represents both the locular tissue and the extreme periphery, the green class represents the union of the outer pericarp and the core of the tomato and the blue class represents the placenta. The black class distribution is not shown because it is attributed to fraction volumes and it has no physical signification. The failure to identify the two other classes may be due to the non-homogeneity of the classes, a phenomena that can be related either to the physiology of the fruit tissues that is not $100 \%$ homogeneous or to the non-spatial homogeneity of the MRI antenna (observable on the $A_{0}$ maps). These points remain open for further studies by adapting the classification algorithm to those phenomena.

\section{CONCLUSION}

In this paper, algorithms for both the reconstruction and the exploitation of relaxtion times and their corresponding amplitude maps from noisy MRI magnitude data were presented. The estimation algorithm showed both stability and accuracy on real and simulated MRI data. The reconstruction was carried out, for the first time as far as our knowledge, on the whole image at once. Furthermore, we were able to plot the $T_{2}$ distribution inside different fruit tissues by using an automatic unsupervised classification technique and to identify characterizing fruit tissues. This work will enable the assessment of fruit characteristics at the subcellular level since the multiple $T_{2}$ are linked with the water status in the different cell structures.

\section{REFERENCES}

[1] H. Adriaensen, M. Musse, S. Quellec, A. Vignaud, M. Cambert, and F. Mariette. MSE-MRI sequence optimisation for measurement of biand tri-exponential T2 relaxation in a phantom and fruit. Magnetic Resonance Imaging, 31(10):1677-1689, 2013.

[2] C. El-Hajj, S. Moussaoui, G. Collewet, and M. Musse. Spatially regularized multi-exponential transverse relaxation times estimation from magnitude magnetic resonance images under Rician noise. In The 26th IEEE International Conference on Image Processing (ICIP), 2019.

[3] E. Hakan and J. A. Fessler. Ordered subsets algorithms for transmission tomography. Physics in Medicine \& Biology, 44(2835-2851), 1999.

[4] J. A. Hartigan and M. A. Wong. Algorithm as 136: A k-means clustering algorithm. Journal of the Royal Statistical Society. Series C (Applied Statistics), 28(1):100-108, 1979.

[5] R. M. Henkelman. Measurement of signal intensities in the presence of noise in mr images. Medical physics, 12(2):232-233, 1985.

[6] D. Hwang and Y. P. Du. Improved myelin water quantification using spatially regularized non-negative least squares algorithm. Journal of Magnetic Resonance Imaging: An Official Journal of the International Society for Magnetic Resonance in Medicine, 30(1):203-208, 2009.

[7] J. Idier. Convex half-quadratic criteria and interacting auxiliary variables for image restoration. IEEE Transactions on Image Processing, 10(7):1001-1009, July 2001.

[8] D. Kumar, T. D. Nguyen, S. A. Gauthier, and A. Raj. Bayesian algorithm using spatial priors for multiexponential $\mathrm{T} 2$ relaxometry from multiecho spin echo MRI. Magnetic resonance in medicine, 68(5):1536-1543, 2012.

[9] C. Laule, I. M. Vavasour, S. H. Kolind, D. K. Li, T. L. Traboulsee, G. W. Moore, and A. L. MacKay. Magnetic resonance imaging of myelin. Neurotherapeutics, 4(3):460-484, 2007.

[10] M. Musse, S. Quellec, M.-F. Devaux, M. Cambert, M. Lahaye, and F. Mariette. An investigation of the structural aspects of the tomato fruit by means of quantitative nuclear magnetic resonance imaging. Magnetic Resonance Imaging, 27(5):709-719, 2009.

[11] J. G. Raya, O. Dietrich, A. Horng, J. Weber, M. F. Reiser, and C. Glaser T2 measurement in articular cartilage: impact of the fitting method on accuracy and precision at low snr. Magnetic Resonance in Medicine: An Official Journal of the International Society for Magnetic Resonance in Medicine, 63(1):181-193, 2010

[12] D. Varadarajan and J. P. Haldar. A Majorize-Minimize framework for Rician and non-central Chi MR images. IEEE transactions on Medical Imaging, 34(10):2191-2202, 2015.

[13] T. Yokoo, Q. Yuan, J. Sénégas, A. J. Wiethoff, and I. Pedrosa. Quantitative R2* mri of the liver with rician noise models for evaluation of hepatic iron overload: Simulation, phantom, and early clinical experience. Journal of Magnetic Resonance Imaging, 42(6):1544-1559, 2015. 